\title{
Single-Component Biohybrid Light-Emitting Diodes Using a White- Emitting Fused Protein
}

Carmen F. Aguiño, ${ }^{\dagger}$ Martina Lang, ${ }^{\ddagger}$ Verónica Fernández-Luna, ${ }^{\dagger}$ Marlene Pröschel, $^{\ddagger}$ Uwe Sonnewald, ${ }^{*}$, Pedro B. Coto, ${ }^{*}, \AA^{\circ}$ and Rubén D. Costa ${ }^{*}+{ }^{\dagger}$

${ }^{\dagger}$ IMDEA Materials Institute, Calle Eric Kandel 2, 28906 Getafe, Madrid, Spain

${ }^{\ddagger}$ Department of Biology, University of Erlangen-Nuremberg, Staudtstr. 5, 91058 Erlangen, Germany

${ }^{\S}$ Department of Physical and Analytical Chemistry, University of Oviedo, Avda. Julián Clavería 8, 33006 Oviedo, Spain

\author{
Supporting Information
}

\begin{abstract}
This work presents a pioneering multidisciplinary approach toward enhancing biohybrid light-emitting diodes (BioHLEDs), merging synthetic biology tools, polymer chemistry, and device engineering to design a thin color downconverting coating with a single white-emitting fluorescent protein (WFP). In particular, the WFP consists of fused red-, green-, and blue-emitting FPs following the so-called protein superglue approach. This WFP shows an efficient and stable white emission originated from a Förster resonance energy transfer between FP moieties. The emission chromaticity is, in addition, easily controlled by the rigidity of the polymer matrix of the coating, reaching photoluminescence quantum yields of $26 \%$ that stand out among intrinsic white-emitting materials. The WFP single-component color downconverting packaging was applied to fabricate BioHLEDs featuring efficient neutral white emission that is stable over $400 \mathrm{~h}$. This represents the most stable BioHLED reported to date. Thus, this work is a landmark for the use of synthetic biology tools to design tailored luminescent biomaterials for lighting applications.
\end{abstract}

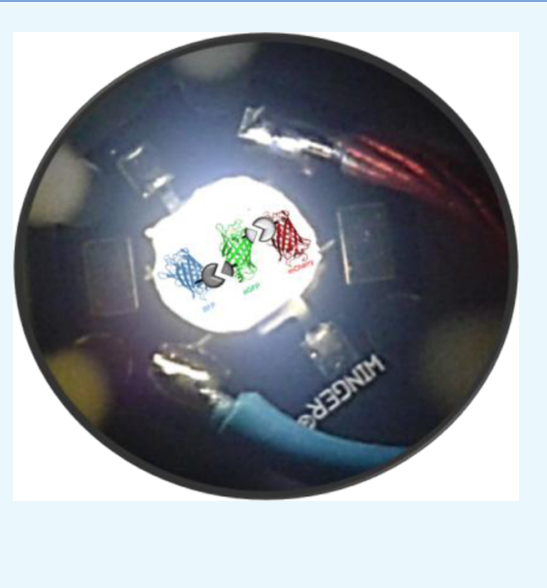

\section{INTRODUCTION}

Over the last years, white biohybrid light-emitting diodes (BioHLEDs) have emerged as a new approach toward sustainable and eco-friendly white lighting sources following the "Green Photonics" concept. ${ }^{1,2}$ Generally speaking, HLEDs use efficient inorganic high-energy emitting chips and fully organic color down-converting packagings to partially convert UV or blue irradiations into the low-energy part of the visible spectrum. The combination of electroluminescence and photoluminescence responses leads to white lighting sources featuring high color quality with $x / y$ CIE coordinates of $0.30-$ $3 / 0.30-3$, color rendering index (CRI) above 80, and correlated color temperatures (CCT) ranging from 2700 to $6500 \mathrm{~K}$. However, these devices exhibit a stability of less than $100 \mathrm{~h}$ caused by the photo- and thermal degradation of the color down-converting materials, ${ }^{1-13}$ which poses a serious limitation for standard applications.

BioHLEDs are a new class of lighting devices that are divided into two families depending on the type of color downconverting packagings. On the one hand, several groups have demonstrated the use of biomaterials, such as cellulose, starch, mucine, and so forth, as matrices to host carbon dots, coordination complexes, laser dyes, and conjugated polymers. ${ }^{9-13}$ The interest in these biomatrices stems from the use of water, the ease of film preparation, and the final mechanical and optical features-for example, transmittance superior to $80 \%$, refractive index between 1 and 1.5 , tensile modulus in the
GPa regimes along with elongations between 1 and 20\%, among others. On the other hand, the use of fluorescent proteins (FPs) as emitters has recently been demonstrated in dry films ${ }^{4,5}$ and in an elastomeric polymer matrix. ${ }^{3,8}$ Although the former achieved energy conversions over $90 \%$ and luminous efficiencies over $100 \mathrm{~lm} / \mathrm{W}$ regardless of the $\mathrm{FP}$ purity grade, the latter showed record stabilities up to $100 \mathrm{~h}$ without degradation of color quality $(\mathrm{CRI}=80)$ and luminous efficiency $(55 \mathrm{~lm} / \mathrm{W})$, as well as a high versatility toward the fabrication of either single-point lighting sources ${ }^{3}$ or microstructured color filters for displays.

Common to the use of FPs is the need of packaging designs to ensure high-quality white light via energy-transfer processes from the UV- or blue-emitting chip to green-emitting FP and from this to the red-emitting FP. In dry films, the major limitations are the control of both the color quality and the luminescence spatial distribution, as FPs are randomly distributed in the films, which implies nonefficient energytransfer processes related to the distance among FPs when the material is collapsed and dry. ${ }^{6}$ In addition, the lack of FP stabilizers limits the device stability. As far as the elastomeric packaging is concerned, two approaches toward white BioHLEDs have been demonstrated, namely millimeter-thick

Received: August 30, 2018

Accepted: October 24, 2018

Published: November 20, 2018 

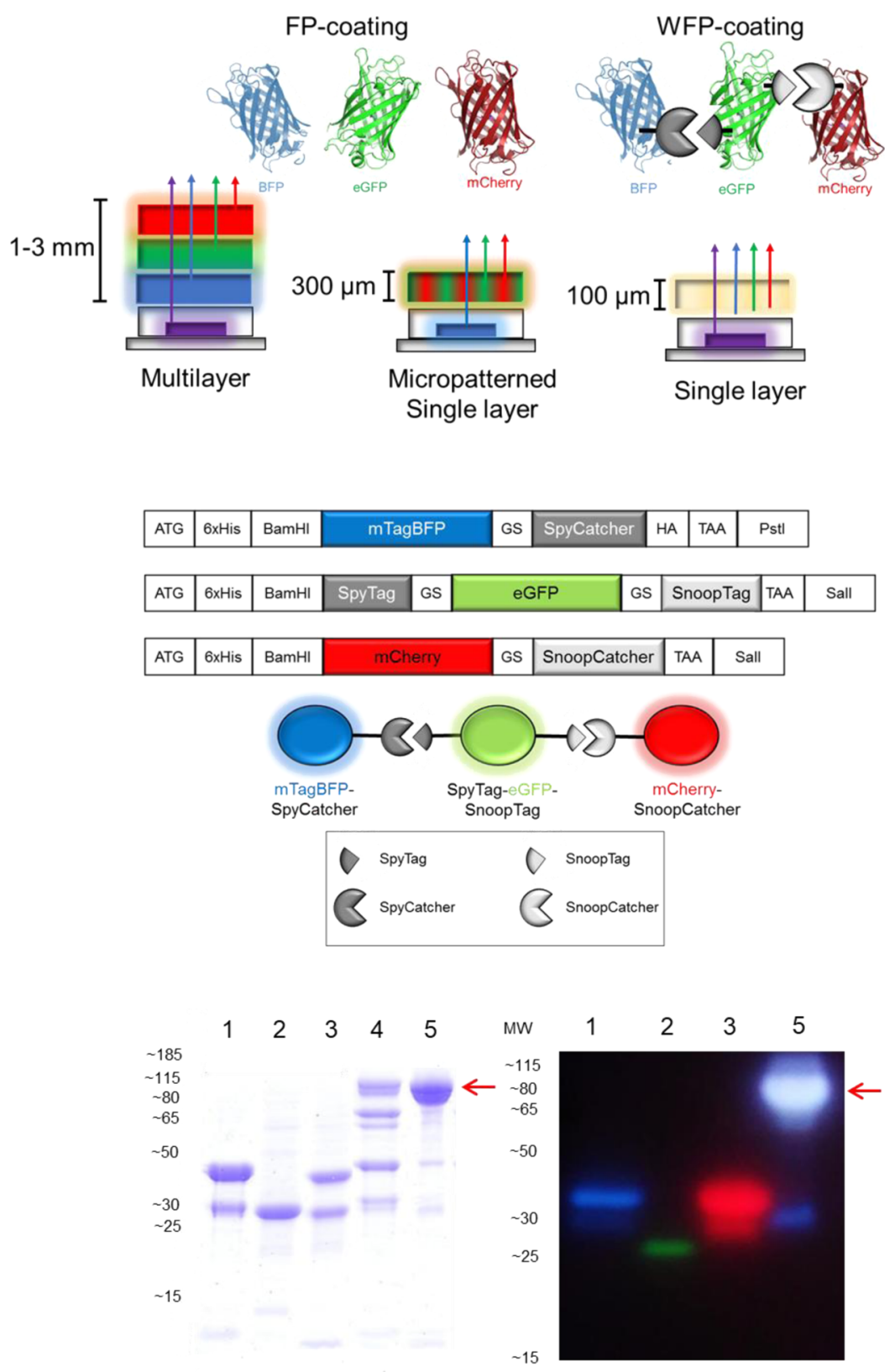

Figure 1. Top: Sketch of different types of BioHLEDs prepared with both FP and WFP coatings. Center: Synthesis of WFP using the protein superglue approach and the schematic representation of the recombinant proteins (top: DNA constructs encoding for the protein components of the WFP complex; bottom: scheme of the WFP/trimeric blue-green-red protein complex mediated by two orthogonal covalent protein superglue pairs, SpyCatcher-SpyTag and SnoopCatcher-SnoopTag). Bottom, left: Coomassie-stained sodium dodecyl sulfate (SDS)-polyacrylamide gel electrophoresis (PAGE) of WFP/trimer single components (lane 1: mTagBFP-SpyCatcher input; lane 2: SpyTag-eGFP-SnoopTag input; lane 3: mCherry-SnoopCatcher input; each $90 \mu \mathrm{M}$ ) and the assembled trimer before (lane 4) and after a second purification step [size exclusion chromatography (SEC), lane 5]. Bottom, right: native SDS-PAGE of the single protein components (lane 1: mTagBFP-SpyCatcher input, lane 2: SpyTag-eGFP-SnoopTag input, lane 3: mCherry-SnoopCatcher input; each $90 \mu \mathrm{M}$ ) and the final SEC-purified and concentrated trimeric form (lane 4) exposed to UV light.

multilayered FP coatings with a bottom-up energy-transfer cascade scheme and micropatterned single-layered FP coatings-Figure $1 .{ }^{3,8}$ The white quality is controlled by the thickness of each layer, the amount of FPs included, and the micropattern design (i.e., line, open, or closed grid). Similar to the dry films approach, the use of three FPs in the elastomeric coating leads to nonreproducible emission features, as the Förster resonance energy-transfer (FRET) process is affected by the random spatial distribution of the FPs going from solution to the rubber stage under vacuum treatment. ${ }^{6}$ An example involving the three FPs is provided in Figure S1. Besides these aspects, the main drawback is the thickness of the packagings (up to millimeters) that leads to poor light outcoupling, associated with both low transmittance and changes in the refractive index caused by the number of interfaces. In addition, the fabrication of both multilayered and micropatterned coatings is tedious, as it involves several deposition/testing steps until the desired white emission is achieved. Hence, it is instrumental to develop thin-film 
coatings based on a single FP emitter, whose photoluminescence leads to white BioHLEDs.

Herein, we took advantage of a new synthetic biology approach based on the protein superglue concept to engineer a novel fused WFP to fabricate single-layered color downconverting packagings for single-component white BioHLEDs. This manuscript discloses three significant advances. First, the synthesis of the first WFP using two fully orthogonal protein superglue systems, SpyCatcher-SpyTag and SnoopCatcherSnoopTag, for covalent and irreversible protein assembly. ${ }^{14-16}$ The Spy and the Snoop systems are widely used posttranslational protein ligation tools based on spontaneous isopeptide bond formation between Catcher and Tag under a wide range of conditions, ${ }^{14}$ but, to the best of our knowledge, this approach has not been applied to FPs yet. In short, blue-, green-, and red-emitting FPs (mTagBFP, eGFP, and mCherry, respectively) were genetically fused to Catcher and Tag domains to ensure proper protein assembly when components are mixed-Figure 1. ${ }^{15,16}$ Second, the optimization of the interaction between the packaging matrix and the WFP leads to stable white emission with photoluminescence quantum yields $(\phi)$ of $26 \%$ that is top among other intrinsic whiteemitting materials such as perovskites, ${ }^{17}$ conjugated polymers, ${ }^{18}$ hybrid nanoparticles, and so forth. ${ }^{19}$ Finally, first single-component BioHLEDs featuring a neutral white color $(\mathrm{CRI}>90)$ stable over hundreds of hours $(>400 \mathrm{~h})$ are presented as a proof of concept of this multidisciplinary approach.

\section{RESULTS AND DISCUSSION}

The preparation of WFPs involved the recombinant expression of the three single FP components of the trimer, mTagBFPSpyCatcher, SpyTag-eGFP-SnoopTag, and mCherry-SnoopCatcher, in Escherichia coli (E. coli M15 [pREP4]) cellsFigures 1 and S2 in the Supporting Information. Subsequent affinity chromatography using Ni-NTA agarose resin under native conditions was applied to purify the FP of interestFigure S3. Having fused cognate Catcher and Tag interaction partners to blue-, green-, and red-emitting FPs, they could be linked irreversibly just by mixing them in an equimolar ratiothat is, in vitro reconstitution; Supporting Information. Covalent interaction kinetics was monitored for up to $24 \mathrm{~h}$ by SDS-PAGE and Western blot analysis-Figure S4. Samples were boiled in a $4 \times$ Laemmli sample buffer containing SDS detergent before gel loading to prove the covalent character of respective Catcher-Tag interactions. ${ }^{14}$ The resulting WFP was then further purified in a second chromatography step to remove monomeric and dimeric side products-Figures 1 and S5. Please notice that the genetic fusion construct was hardly expressed in E. coli and probably quite unstable as shown by the SDS analysis after purification.

Pure WFP was checked for fluorescent properties by performing a native SDS-PAGE followed by UV light exposure-Figures 1 and S5. In more detail, the steady-state absorption and photoluminescence features of the purified WFP in solution confirm the success of protein binding in a trimeric form-Figure 2. The absorption spectrum consists of four well-defined bands centered at 340, 400, 490, and $585 \mathrm{~nm}$, resulting from the sum of the absorption features of each FP moiety - that is, mTagBFP, eGFP, and mCherry. However, photoluminescence is heavily dependent on the excitation wavelength $\left(\lambda_{\text {exc }}\right)$. In particular, excitation between 300-310 and $340-360 \mathrm{~nm}$ leads to a white emission with three peaks
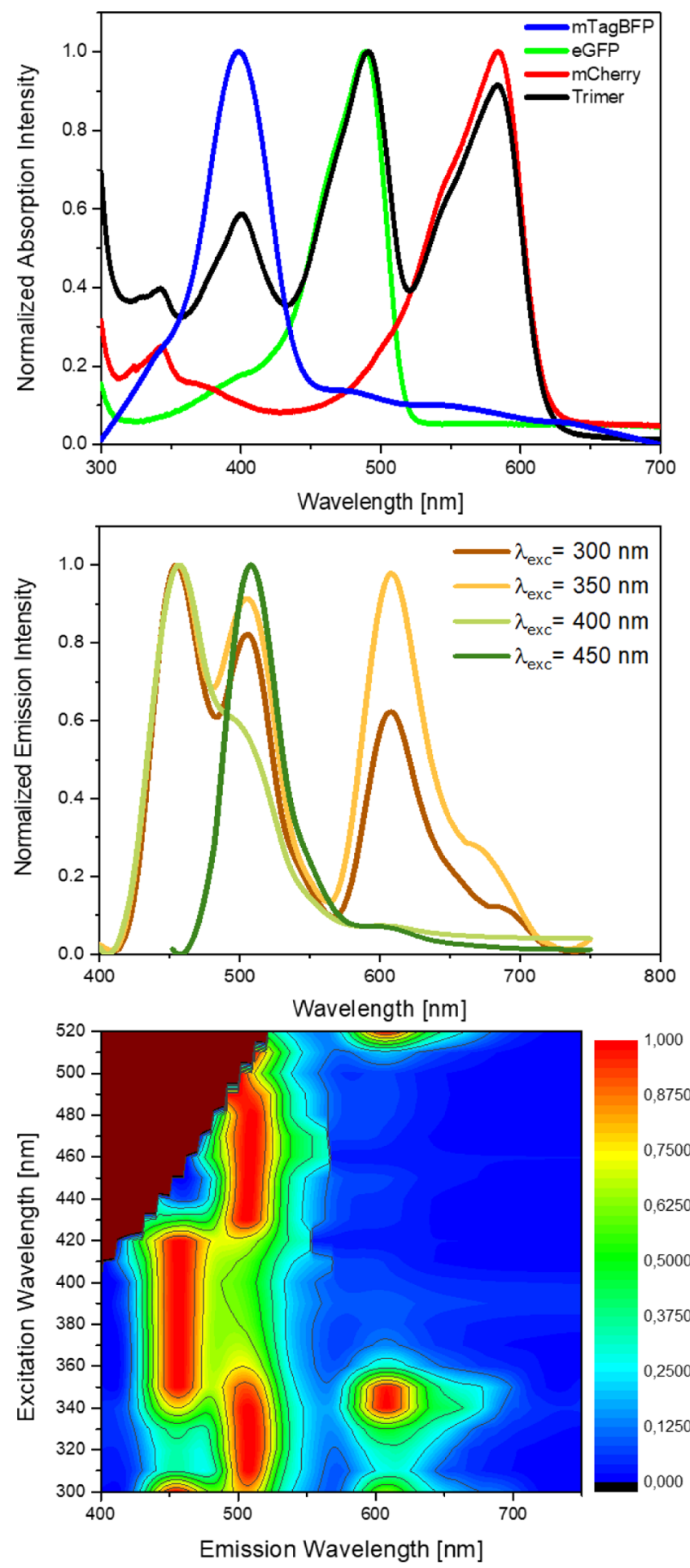

Figure 2. Normalized steady-state absorption (top) and emission spectra (center), as well as excitation wavelength vs emission wavelength map (bottom) of the WFP in aqueous solution.

located at 450, 510, and $610 \mathrm{~nm}$ and with a balanced emission intensity, whereas excitation between $310-330 \mathrm{~nm}$ and $360-$ $400 \mathrm{~nm}$ leads to emission bands with the same peaks, but with dominant intensity for the green and blue peaks, respectively. As mCherry features an absorption band centered at $340 \mathrm{~nm}$, the photoluminescence behavior suggests that the FRET process from mTagBFP to eGFP is effective at $\lambda_{\text {exc }}<350 \mathrm{~nm}$, but not from eGFP to mCherry. Indeed, exclusive excitation of 
the eGFP moiety $\left(\lambda_{\text {exc }}\right.$ in the range of $450-500 \mathrm{~nm}$ ) does not lead to significant activation of the mCherry emission via FRET-Figure 2. This might be related to both the nature of the interaction systems used to fuse the single components (Spy and Snoop) and the ease of conformational changes of the WFP in solution. There are certain differences in the binding units used for the linkage of eGFP-mCherry (Snoopsystem) and the mTagBFP-eGFP (Spy-system). Both systems mainly differ in their amino acid composition and structure, ${ }^{14-16}$ which might affect the energy transfer. Another possibility could be that the fusion of interaction domains to the FPs and the linkage reaction alters the stability of mCherry, eGFP, and mTagBFP moieties.

As far as the structural flexibility of WFP in solution is concerned, it is well-known that the FRET process in oligomeric FPs can be enhanced in solid matrices either increasing its concentration ${ }^{20-22}$ or locking the structure of oligomeric FPs. ${ }^{23,24}$ Following this rationale, we investigated the photoluminescence features of novel WFP in an elastomeric polymer matrix, whose mechanical and optical features are suitable for LED packaging, while it has been proven that the FP emission is significantly stabilized under storage conditions. ${ }^{1-3,8}$ In particular, the elastomeric matrix consists of a mix of branched and linear poly(ethylene oxide) (l-PEO) compounds-that is, trimethylolpropane ethoxylate (TMPE) and l-PEO, respectively-in a WFP solution (0.1 $\mathrm{mg} / \mathrm{mL}$ ). This is followed by (i) vigorous stirring for a few hours forming a hydrogel and (ii) a drying process under gentle vacuum overnight forming the rubberlike material-see Supporting Information.

As a first step, WFP was embedded in thin rubbers $(50 \mu \mathrm{m})$ with a TMPE/l-PEO mass ratio of $12: 1$ and the photoluminescence features were studied at $\lambda_{\mathrm{exc}}=400 \mathrm{~nm}$, which corresponds to the maximum absorption of mTagBFP. Similar to the emission of WFP in solution $(0.1 \mathrm{mg} / \mathrm{mL}), 12: 1$ rubbers show an emission band composed of three peaks centered at 450,510 , and $610 \mathrm{~nm}$. However, the intensity of both the green- and red-emitting peaks is significantly enhanced, while the intensity of the blue-emitting part is correspondingly reduced. This is in line with the enhancement of the FRET process between FPs in solid-state and/or rigid matrices. ${ }^{19-24}$ Recently, we have shown that the rheological features of the hydrogels can be easily controlled by the molecular weight and the amount of 1-PEO used to prepare micropatterned coatings. ${ }^{8}$ In the same manner, it is also possible to fine-tune the rigidity of the matrix network in the final rubber by increasing the amount of 1-PEO. This is nicely confirmed by dynamic rheological assays, in which the mechanical modulus increases. For example, if $G^{\prime}$ at $1 \mathrm{rad} / \mathrm{s}$ is compared, the values obtained for $12: 1,8: 1,6: 1$, and $4: 1$ rubbers are $26.2,35.7,58.3$, and $80.6 \mathrm{kPa}$, respectively-Figure S6. Figure 3 displays the changes of the emission spectra upon increasing the rigidity of the rubbers. Here, the intensity balance between green and red emission peaks is kept, while the blue emission peak steadily decreases. Among all the polymer matrix compositions, 6:1 rubbers show the best white emission in terms of color quality - that is, $x / y \mathrm{CIE}$ color coordinates of $0.34 / 0.37, \mathrm{CRI}=$ 93, and CCT of $4900 \mathrm{~K}-$ Figure 3. In addition, the $\phi$ of the $6: 1$ rubber was $26 \%$, representing a record value in intrinsic white-emitting materials. ${ }^{17-19}$ Finally, the emission features are stable under storage (room temperature and moisture) and stress (temperature increase from 30 to $90{ }^{\circ} \mathrm{C}$ with a ramp of 1 ${ }^{\circ} \mathrm{C} / \mathrm{min}$ ) conditions-Figure $\mathrm{S} 7$.
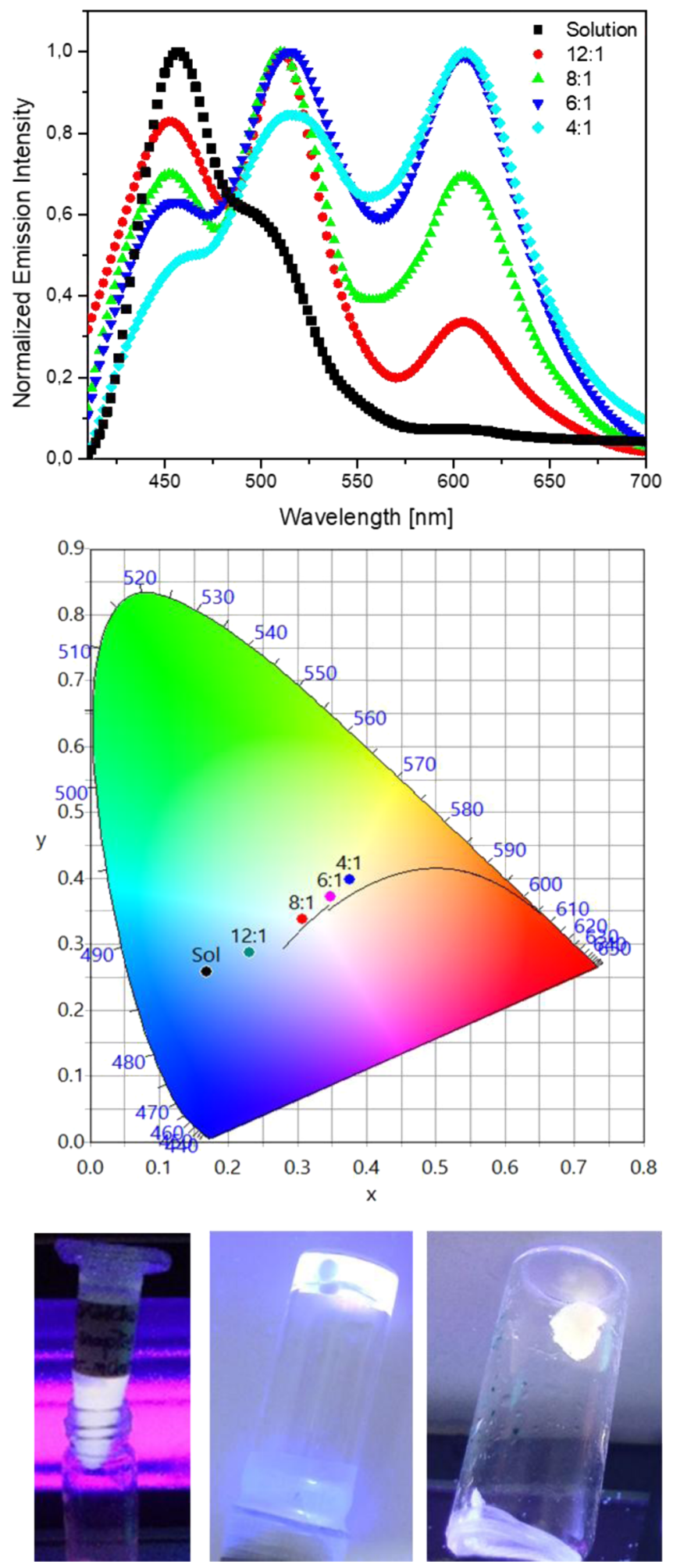

Figure 3. Changes of the emission spectra (top) and the $x / y$ CIE color coordinates (center) of rubbers with the WFP embedded into different TMPE/l-PEO mass ratios of the network matrix $\left(\lambda_{\text {exc }}=400\right.$ $\mathrm{nm}$ ). Pictures of WFP (bottom) in aqueous solution (left), hydrogel (middle), and 6:1 rubber (right).

Encouraged by these results, we decided to fabricate BioHLEDs using commercial UV-LEDs with a $400 \mathrm{~nm}$ emitting chip-that is, WINGER WEPUV3-S2 UV Power LED Star (Blacklight) $1.2 \mathrm{~W}$-covered by a thin $(100 \mu \mathrm{m})$ 
color down-converting coating based on 6:1 rubbers with the novel WFP (5 mg)-Figure 1. The color conversion and stability of the devices were characterized at different applied currents and constant applied current, respectively. Changes in the emission spectrum, luminous efficiency, and temperature of the BioHLEDs were monitored-see Supporting Information. Upon applying different currents ranging from 20 to $200 \mathrm{~mA}$, the color down-conversion efficiency $\left(\eta_{\text {con }}\right)$, which is defined as the ratio between the area of the LED emission covered with a coating with and without the WFP, holds constant at around $75 \%$-Figure S8, indicating that there is no saturation, bleaching, and/or nonlinear quenching effects upon increasing the driving current. For the stability studies, we first selected the applied current at which the maximum efficiency of the commercial LED is achieved-that is, $10 \mathrm{~mA}$, from which this value exponentially decreases because of the increase in the temperature of the emitting chip caused by the reduction of the internal quantum efficiency-Figure S9. As such, we decided to monitor the color quality and device efficiency (15 $\mathrm{lm} / \mathrm{W}$ ) at this applied current over time-Figure 4. Remarkably, the single-component white BioHLEDs featured a broad emission spectrum consisting of four peaks centered at $400 \mathrm{~nm}$ (LED emission) and 450, 520, and $610 \mathrm{~nm}$ (color down-conversion coating). This light spectrum corresponds to a neutral white emission with $x / y$ CIE color coordinates of $0.37 / 0.38, \mathrm{CRI}=91$, and CCT of $4300 \mathrm{~K}^{25}$ Moreover, the BioHLEDs showed minimal changes in the emission spectrum at different measured angles-Figure S10, suggesting a uniform spherical light distribution. For comparison purposes, this emission spectrum is similar to that of the direct sunlight measured in June 2018 at noon in Madrid (Spain). Finally, both the color quality and luminous efficiency hold constant over $200 \mathrm{~h}$ under continuous operation-Figure 4. From this point on, the red-emitting part of the spectrum starts to attenuate, changing the emission color toward a cold white emission and decreasing the luminous efficiency to $10 \mathrm{~lm} / \mathrm{W}$. These features hold constant for another $200 \mathrm{~h}(<5 \%$ change)-Figure 4, representing one of the most stable BioHLEDs reported so far. ${ }^{1-5,8}$ The loss of the red-emitting part is ascribed to the degradation of the mCherry protein. ${ }^{26}$ This is in line with previous studies, which showed that its nonradiative deactivation via a dark excited state is faster than those of the high-energy emitting analogous. In addition, this deactivation process enhances the mCherry absorption intensity at the high-energy part of the spectrum, further reducing the emission from the other FPs, affecting the overall efficiency of the BioHLED. ${ }^{8}$

\section{CONCLUSIONS}

In conclusion, this work presents the preparation of a novel white-emitting fused FP applying the protein superglue approach to $\mathrm{mTagBFP}$, eGFP, and mCherry moieties and its implementation in solid-state lighting. This novel WFP shows an excellent stability in an elastomeric matrix used for color down-converting purposes in HLEDs, such as BioHLEDs. It is worth noting that the emission spectra of WFP is easily tuned by changing the rigidity of the polymer network, leading to a neutral white emission associated with a record $\phi$ of $26 \%$ that is, in addition, stable for device applications. This allows us to prepare thin, single-component white packagings that circumvent the efficiency limits and fabrication concerns of current FP-based color down-converting packagings used in BioHLEDs (i.e., thick multilayered or thin microstructured
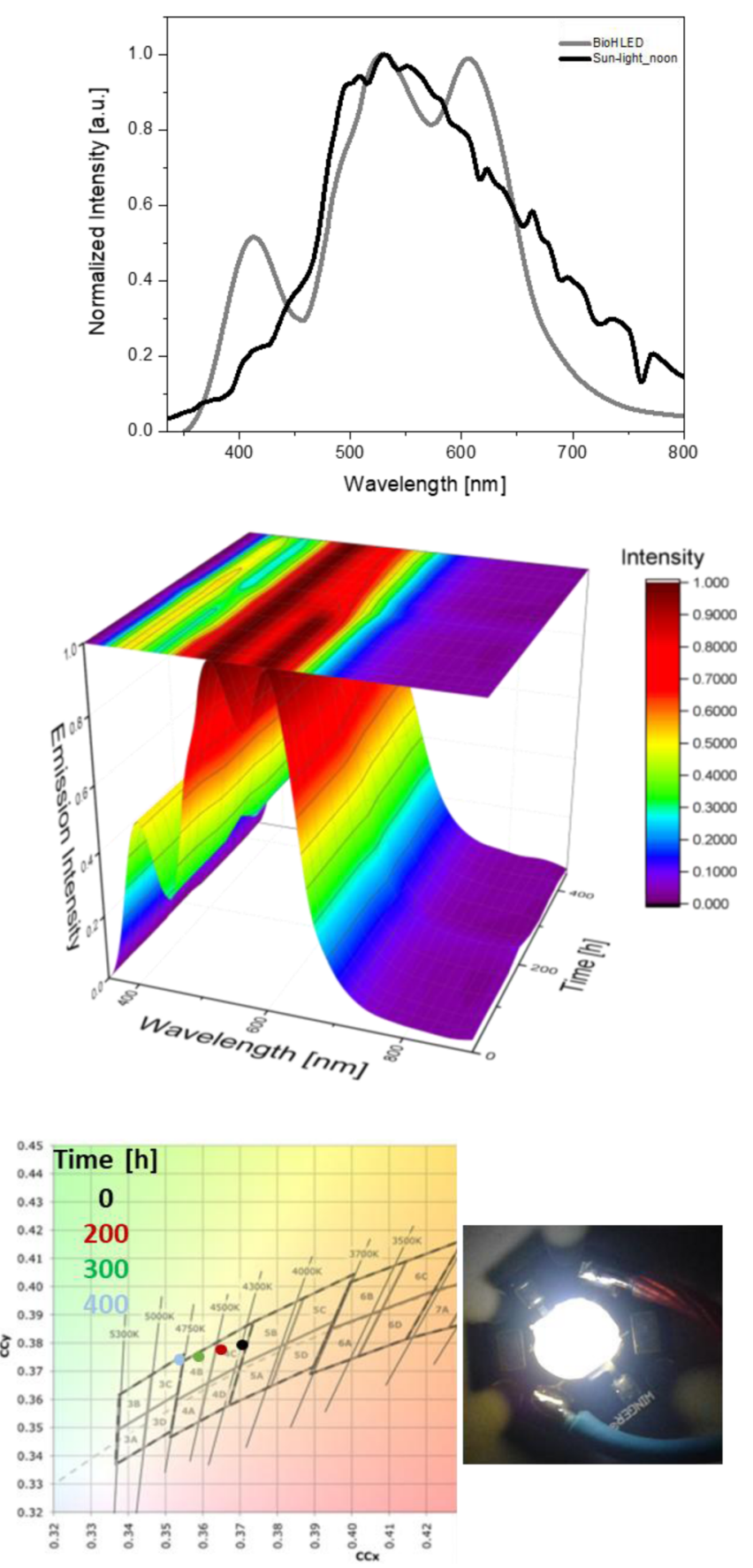

Figure 4. Top: Emission spectra of BioHLED and direct sunlight measured in June 2018 at noon in Madrid (Spain). Center: Emission $3 \mathrm{D}$ plot showing the changes of BioHLED emission spectra over time under continuous operation. Bottom: Changes of the $x / y$ CIE color coordinates of the BioHLED over time under continuous operation (left) and a picture of a BioHLED under continuous operation conditions (right).

configurations). As a proof of concept, BioHLEDs prepared using this novel WFP packaging featured efficiencies ranging from 10 to $15 \mathrm{~lm} / \mathrm{W}$ in concert with a neutral white emission stable over $400 \mathrm{~h}$. Subsequent changes in the device performance (efficiency and color) are associated with the degradation of the red-emitting protein (mCherry), pointing out the need to enhance photostability of red-emitting FPs, which could be done by simply exchanging mCherry with 
another red FP that shows more suitable properties. Possible candidates for the exchange could be the FPs mRuby ${ }^{27}$ and mScarlet. ${ }^{28}$ Work along these lines is currently underway in our laboratories. This work represents a landmark for the use of synthetic biology tools to design tailored luminescent biomaterials for lighting applications.

\section{EXPERIMENTAL SECTION}

4.1. Cloning of Gene Constructs. To combine the genes encoding for the different superglue protein domains (SpyCatcher, SpyTag, SnoopCatcher, and SnoopTag) and the FPs (eGFP, mTagBFP, and mCherry), the overlapextension polymerase chain reaction (PCR) method was performed using gene-specific oligonucleotides (Tables S1 and S2). mTagBFP5 was genetically fused to SpyCatcher, the eGFP6-DNA construct was constructed to be surrounded by SpyTag-peptide N-terminally and SnoopTag at the Cterminus. The red FP mCherry7 was fused to the SnoopCatcher domain. FPs and Catcher-/Tag-domains were separated by a linear, flexible glycine-serine linker (GSGSGSG) increasing the probability for proper post-translational protein folding (sequences of final gene constructs are attached). The cloning scheme and primers used are listed in Tables S1 and S2. High-Fidelity Phusion DNA-polymerase (Thermo Fisher Scientific) was used to perform all PCR amplifications. After overlap-PCR and gel extraction following the manufacturer's instruction (QIAquick Gel Extraction Kit, Qiagen), the desired DNA products were ligated into the PQE-9 E. coli expression vector (Qiagen) containing an N-terminal 6xHis affinity tag, using compatible sticky ends (restriction enzymes from Thermo Fisher Scientific) and T4 DNA ligase (Thermo Fisher Scientific). Subsequent transformation into XL1Blue chemically competent $E$. coli cells and plasmid isolation were performed. The sequences of all pQE-9 expression constructs were verified using Sanger Sequencing (GATC Biotech). Recombinant protein expression and purification: the pQE-9 expression constructs containing the gene of interest $\mathrm{N}$ terminally fused to the His6 tag encoded in the pQE-9 vector, were transformed into chemically competent M15 [pREP4] E. coli cells (Qiagen), and plated on LB agar plates with $200 \mu \mathrm{g} /$ $\mathrm{mL}$ ampicillin and $25 \mu \mathrm{g} / \mathrm{mL}$ kanamycin. Single colonies were grown overnight at $28^{\circ} \mathrm{C}$ in liquid LB medium ( $10 \mathrm{~g} \mathrm{NaCl}, 10$ g Trypton, 5 g yeast extract per $1 \mathrm{~L})$ containing $25 \mu \mathrm{g} / \mathrm{mL}$ kanamycin and $200 \mu \mathrm{g} / \mathrm{mL}$ ampicillin. The overnight cultures were used to inoculate $1 \mathrm{~L}$ expression cultures (LB medium with the appropriate antibiotics: $25 \mu \mathrm{g} / \mathrm{mL}$ kanamycin and 200 $\mu \mathrm{g} / \mathrm{mL}$ ampicillin) to an optical density of 0.2 at $600 \mathrm{~nm}$. The expression cultures were grown with shaking at 180-200 rpm at $28{ }^{\circ} \mathrm{C}$ until an optical density $\mathrm{OD}_{600}$ of 0.5 was reached. By adding $1 \mathrm{mM}$ IPTG (Roth) (final concentration), recombinant protein expression was induced for $4 \mathrm{~h}$ at $28{ }^{\circ} \mathrm{C}$ with shaking at $180-200 \mathrm{rpm}$ prior to harvest by centrifugation $(5000 \mathrm{~g}, 20$ min, $4{ }^{\circ} \mathrm{C}$ ). Cell pellets were frozen and stored at $-20{ }^{\circ} \mathrm{C}$ until further processing.

For protein purification via affinity chromatography, harvested cell pellets were thawed on ice and resuspended in lysis buffer containing Pefabloc protease inhibitor (Roth) (1 $\mathrm{mM}$ final concentration) and lysozyme $(1 \mathrm{mg} / \mathrm{mL}$ final concentration) to minimize protein degradation and increase lysis efficiency.

Cell lysis was further performed by sonication on ice ( $\operatorname{six} 10$ $\mathrm{s}$ bursts with cooling pause between each burst). The lysate was then centrifuged at $10000 \mathrm{~g}$ for $30 \mathrm{~min}$ at $4{ }^{\circ} \mathrm{C}$ to separate the soluble (supernatant) from the insoluble (pellet) proteins. Recombinant proteins from the supernatant, containing an $\mathrm{N}$ terminal His6 tag, were purified using nickel nitrilotriacetic acid (Ni-NTA; Qiagen) affinity chromatography under native (nondenaturing) conditions following the manufacturer's instructions (Qiagen Expressionist). ${ }^{8}$ The following buffers were used: lysis buffer $\left(50 \mathrm{mM} \mathrm{NaH} \mathrm{PO}_{4}, \mathrm{pH} 8.0 ; 300 \mathrm{mM}\right.$ $\mathrm{NaCl}$; and $250 \mathrm{mM}$ imidazole). Polypropylene columns (5 $\mathrm{mL}$; Qiagen) packed with Ni-NTA Agarose resin (Qiagen) were used for purification. Purified proteins were dialyzed in $1 \times$ PBS buffer (136 mM NaCl, $2.7 \mathrm{mM} \mathrm{KCl,} 8 \mathrm{mM} \mathrm{Na}_{2} \mathrm{HPO}_{4}$, and $\left.1.8 \mathrm{mM} \mathrm{KH} \mathrm{KO}_{4}, \mathrm{pH} 7.4\right)$. The concentration of the recombinant proteins was determined by measuring the absorption at $280 \mathrm{~nm}$ using a Nano-Drop Spectrophotometer ND-1000 (Peqlab) and taking into account the specific molar extinction coefficients of the proteins. If needed, proteins were concentrated using Amicon Ultra centrifugal filter units with $10 \mathrm{kDa}$ MWCO (Merck, Millipore). In vitro interaction of single-protein components by split isopeptide reconstitution: purified fluorescent fusion proteins were mixed in an equimolar ratio $(90 \mu \mathrm{M}$ input each, resulting in a $30 \mu \mathrm{M}$ final concentration each) and incubated for $24 \mathrm{~h}$ at $4{ }^{\circ} \mathrm{C}$ while shaking at $500 \mathrm{rpm}$. Spontaneous isopeptide bond formation was monitored by taking samples every hour and performing interaction kinetics (Figure S4). 4× Laemmli buffer (200 mM Tris- $\mathrm{HCl}, \mathrm{pH} 6.8,18 \% \beta$-mercaptoethanol, $40 \%$ glycerol, $0.01 \%$ bromophenol blue, and $8 \%$ SDS) was added to the samples prior to boiling at $95{ }^{\circ} \mathrm{C}$ for $10 \mathrm{~min}$. Proteins were separated on SDS-PAGE (12\% Bis-Tris gel) and either directly stained with Coomassie Brilliant Blue or transferred to a nitrocellulose membrane (Anti-His peroxidase linked Western blot analysis) to visualize the formation and content of covalent products (dimers and trimers) resulting from the interaction between tag and catcher constructs over time.

SEC of the trimer: $24 \mathrm{~h}$ after starting the covalent reaction, the interaction mixture was subjected to SEC to separate monomers and dimers and to isolate the desired trimer. Prior to loading the proteins on the column, the solution was concentrated up to $10 \mathrm{mg}$ protein/mL using Amicon Ultra centrifugal filter units $(100 \mathrm{kDa})$. A Superdex 200 Prep Grade $16 / 60$ column was used with $50 \mathrm{mM} \mathrm{PO}_{4}{ }^{2-}, 150 \mathrm{mM} \mathrm{NaCl}$, $\mathrm{pH} 7.0$ as running buffer. The SEC fractions were monitored on SDS-PAGE, fractions containing the pure trimer were pooled and concentrated to desired concentrations for further LED application.

4.2. Preparation of WFP-Based Rubbers. As a first step, the buffer solution with WFP was added to a mixture of branched and 1-PEO compounds-that is, TMPE with Mn of $450 \mathrm{~mol} \mathrm{wt}$ and 1-PEO with $\mathrm{Mn}$ of $5 \times 10^{6} \mathrm{~mol} \mathrm{wt}$, respectively. Both materials were purchased from SigmaAldrich and used as received. The initial hydrogel is prepared by adding TMPE (100 mg) to the WFP aqueous solution under stirring conditions ( $500 \mathrm{rpm}$ overnight). At this point, different amounts of 1-PEO were added and further stirred for $24 \mathrm{~h}$. Finally, the gels were placed into a mold and dried under gentled vacuum (1 mbar) over night.

4.3. Spectroscopic and Mechanical Characterizations. The steady-state absorption and emission measurements were performed in both solution and thin film coated on a quartz substrate using UV-2600 Spectrometer (Shimadzu) and FS5 Spectrofluorometer (Edinburgh) coupled with integrating sphere for $\phi$ measurements. Thermal stability was obtained using a heating station coupled to (i) an IR camera (FLIR 
FS5), (ii) an excitation source of LED array $(4 \times 390$ EDs $)$, and (iii) a detection station consisting of spectrophotometer (Avaspec-ULS2048L-USB2). The thickness was measured using an Alpha-Step D500 Profilometer (Tencor) and further analyzed by using Gaussian functions as implemented in the software. The rheological behavior of the rubbers was measured under oscillatory mode using a parallel plate rheometer (AR200EX, TA Instruments) with disposable aluminum plates. Samples of $25 \mathrm{~mm}$ in diameter and around $0.8 \mathrm{~mm}$ of thickness were placed between the plates of the rheometer and subjected to an oscillatory shear strain of constant amplitude of $5 \%$ and a fixed normal force of $10 \mathrm{~N}$, during a frequency sweep at $30{ }^{\circ} \mathrm{C}$.

4.4. Fabrication and Characterization of BioHLEDs. UV-LED WINGER WEPUV3-S2 UV Power LED Star (Blacklight) $1.2 \mathrm{~W}$ was purchased and modified with the WFP-coating. The preparation of BioHLEDs concerns a twostep procedure. First, the encapsulation system of the LED was coated with the WFP hydrogel. Second, LED is placed in the vacuum station for drying under 1 mbar overnight. The amount of WFP was optimized to realize a white emission upon increasing the applied current as mentioned in the text. The BioWHLEDs were characterized using Keithley 2400 as a current source, while the luminous efficiency and changes of the electroluminescence spectrum were monitored using an Avantes spectrophotometer (Avaspec-ULS2048L-USB2) in conjunction with an integrating sphere (Avasphere 30-Irrad). At the applied driving conditions, the top temperature of LED did not significantly change from room-temperature values over time.

\section{ASSOCIATED CONTENT}

\section{S Supporting Information}

The Supporting Information is available free of charge on the ACS Publications website at DOI: 10.1021/acsomega.8b02226.

Preparation and characterization of the white-emitting fused proteins and spectroscopic and rheological characterization of the down-converting coating (PDF)

\section{AUTHOR INFORMATION}

\section{Corresponding Authors}

*E-mail: uwe.sonnewald@fau.de (U.S.).

*E-mail: branapedro@uniovi.es (P.B.C.).

*E-mail: ruben.costa@imdea.org (R.D.C.).

\section{ORCID $\odot$}

Pedro B. Coto: 0000-0001-9199-8387

Rubén D. Costa: 0000-0003-3776-9158

\section{Author Contributions}

Carmen F. Aguiño and Martina Lang contributed equally to this work. The manuscript was written through contributions from all authors. All authors have given approval to the final version of the manuscript.

\section{Notes}

The authors declare no competing financial interest.

\section{ACKNOWLEDGMENTS}

V.F-L., C.F.A., and R.D.C. acknowledge the program "Ayudas para la atracción de talento investigador-Modalidad 1 of the Consejeríade Educación, Juventud y Deporte-Comunidad de Madrid with reference number 2016-T1/IND-1463”. R.D.C. acknowledges the Ramón y Cajal program from the Spanish MINECO (RYC-2016-20891) and the 2018 Leonardo Grant for Researchers and Cultural Creators from BBVA Foundation. M.L., M.P., and U.S. were supported by the Emerging Field Initiative of FAU (project: SynBio). P.B.C. acknowledges financial support from the Regional Council for Industry and Employment of the Principality of Asturias and the European Regional Development Fund (ERDF). We would like to thank Dr. Jörg Hofmann for the help with the SEC of proteins.

\section{REFERENCES}

(1) Fernández-Luna, V.; Coto, P. B.; Costa, R. D. When Fluorescent Proteins Meet White Light-Emitting Diodes. Angew. Chem., Int. Ed. 2018, 57, 8826-8836.

(2) Fresta, E.; Fernández-Luna, V.; Coto, P. B.; Costa, R. D. Merging Biology and Solid-State Lighting: Recent Advances in Lightemitting Diodes Based on Biological Materials. Adv. Funct. Mater. 2018, 28, 1707011.

(3) Weber, M. D.; Niklaus, L.; Pröschel, M.; Coto, P. B.; Sonnewald, U.; Costa, R. D. Bioinspired Hybrid White Light-Emitting Diodes. Adv. Mater. 2015, 27, 5493-5498.

(4) Nizamoglu, S. Fluorescent Proteins For Color-Conversion Lightemitting Diodes. SDÜ Fen Bil Enst Der. 2016, 20, 490.

(5) Press, D. A.; Melikov, R.; Conkar, D.; Firat-Karalar, E. N.; Nizamoglu, S. Fluorescent Protein Integrated White LEDs for Displays. Nanotechnology 2016, 27, 45LT01.

(6) Niklaus, L.; Dakhil, H.; Kostrzewa, M.; Coto, P. B.; Sonnewald, U.; Wierschem, A.; Costa, R. D. Easy and Versatile Coating Approach for Long-living White Hybrid Light-emitting Diodes. Mater. Horiz. 2016, 3, 340-347.

(7) Reddy, M. S. P.; Park, C. Bright Luminescence from Pure DNAcurcumin-based Phosphors for Biohybrid Light-emitting Diodes. Sci. Rep. 2016, 6, 32306.

(8) Niklaus, L.; Tansaz, S.; Dakhil, H.; Weber, K. T.; Pröschel, M.; Lang, M.; Kostrzewa, M.; Coto, P. B.; Detsch, R.; Sonnewald, U.; Wierschem, A.; Boccaccini, A. R.; Costa, R. D. Micropatterned DownConverting Coating for White Bio-Hybrid Light-Emitting Diodes. Adv. Funct. Mater. 2017, 27, 1601792.

(9) Benson, N.; Ghimire, A.; Pattammattel, A.; Kumar, C. V. Protein Biophosphors: Biodegradable, Multifunctional, Protein-Based Hydrogel for White Emission, Sensing, and pH Detection. Adv. Funct. Mater. 2018, 27, 1702955.

(10) Zhou, D.; Zou, H.; Liu, M.; Zhang, K.; Sheng, Y.; Cui, J.; Zhang, H.; Yang, B. Surface Ligand Dynamics-Guided Preparation of Quantum Dots-Cellulose Composites for Light-Emitting Diodes. ACS Appl. Mater. Interfaces 2015, 7, 15830-15839.

(11) Tetsuka, H.; Nagoya, A.; Asahi, R. Highly Luminescent Flexible Amino-functionalized Graphene Quantum Dots@Cellulose Nanofiber-clay Hybrids for White-light Emitting Diodes. J. Mater. Chem. C 2015, 3, 3536-3541.

(12) Sun, M.; Qu, S.; Hao, Z.; Ji, W.; Jing, P.; Zhang, H.; Zhang, L.; Zhao, J.; Shen, D. Towards Efficient Solid-state Photoluminescence Based on Carbon-nanodots and Starch Composites. Nanoscale 2014, 6, 13076-13081.

(13) Hendler, N.; Belgorodsky, B.; Mentovich, E. D.; Gozin, M.; Richter, S. Efficient Separation of Dyes by Mucin: Toward Bioinspired White-Luminescent Devices. Adv. Mater. 2011, 23, 4261-4264.

(14) Zakeri, B.; Fierer, J. O.; Celik, E.; Chittock, E. C.; SchwarzLinek, U.; Moy, V. T.; Howarth, M. Peptide Tag Forming a Rapid Covalent Bond to a Protein, Through Engineering a Bacterial Adhesin. Proc. Natl. Acad. Sci. U.S.A. 2012, 109, E690-E697.

(15) Veggiani, G.; Nakamura, T.; Brenner, M. D.; Gayet, R. V.; Yan, J.; Robinson, C. V.; Howarth, M. Programmable Polyproteams Built using Twin Peptide Superglues. Proc. Natl. Acad. Sci. U.S.A. 2016, 113, 1202-1207. 
(16) Reddington, S. C.; Howarth, M. Secrets of a Covalent Interaction for Biomaterials and Biotechnology: SpyTag and SpyCatcher. Curr. Opin. Chem. Biol. 2015, 29, 94-99.

(17) Bidikoudi, M.; Fresta, E.; Costa, R. D. White Perovskite Based Lighting Devices. Chem. Commun. 2018, 54, 8150-8169.

(18) Ying, L.; Ho, C.-L.; Wu, H.; Cao, Y.; Wong, W.-Y. White Polymer Light-Emitting Devices for Solid-State Lighting: Materials, Devices, and Recent Progress. Adv. Mater. 2014, 26, 2459-2473.

(19) Ezquerro, C.; Fresta, E.; Serrano, E.; Lalinde, E.; GarcíaMartínez, J.; Berenguer, J. R.; Costa, R. D. White-Emitting Organometallo-Silica Nanoparticles for Sun-like Light-emitting Diodes. Mater. Horiz. 2018, DOI: 10.1039/c8mh00578h.

(20) Gather, M. H.; Yun, S. H. Bio-optimized Energy Transfer in Densely Packed Fluorescent Protein Enables Near-maximal Luminescence and Solid-State Lasers. Nat. Commun. 2014, 5, 5722.

(21) Zajac, J. M.; Schubert, M.; Roland, T.; Keum, C.; Samuel, I. D. W.; Gather, M. C. Time-Resolved Studies of Energy Transfer in Thin Films of Green and Red Fluorescent Proteins. Adv. Funct. Mater. 2018, 28, 1706300.

(22) Dietrich, C. P.; Siegert, M.; Betzold, S.; Ohmer, J.; Fischer, U.; Höfling, S. Exciton Dynamics in Solid-State Green Fluorescent Protein. Appl. Phys. Lett. 2017, 110, 043703.

(23) Costantini, L. M.; Fossati, M.; Francolini, M.; Snapp, E. L. Assessing the Tendency of Fluorescent Proteins to Oligomerize Under Physiologic Conditions. Traffic 2012, 13, 643-649.

(24) Vámosi, G.; Mücke, N.; Müller, G.; Krieger, J. W.; Curth, U.; Langowski, J.; Tóth, K. EGFP Oligomers as Natural Fluorescence and Hydrodynamic Standards. Sci. Rep. 2016, 6, 33022.

(25) Colorimetry: Understanding the CIE System; Schanda, J., Ed.; Wiley Interscience, 2007.

(26) Pröschel, M.; Kraner, M. E.; Horn, A. H. C.; Schäfer, L.; Sonnewald, U.; Sticht, H. Probing the Potential of CnaB-type Domains for The Design of Tag/catcher Systems. PLoS One 2017, 12, No. e0179740.

(27) Kredel, S.; Oswald, F.; Nienhaus, K.; Deuschle, K.; Röcker, C.; Wolff, M.; Heilker, R.; Nienhaus, G. U.; Wiedenmann, J. mRuby, a Bright Monomeric Red Fluorescent Protein for Labeling of Subcellular Structures. PLoS One 2009, 4, No. e4391.

(28) Bindels, D. S.; Haarbosch, L.; van Weeren, L.; Postma, M.; Wiese, K. E.; Mastop, M.; Aumonier, S.; Gotthard, G.; Royant, A.; Hink, M. A.; Gadella, T. W. J. mScarlet: a Bright Monomeric Red Fluorescent Protein for Cellular Imaging. Nat. Methods 2017, 14, 5356. 